\title{
Brains striving for coherence: Long-term cumulative plot formation in the default mode network
}

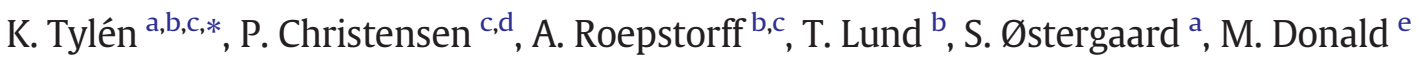 \\ a Center for Semiotics, Department for Aesthetics and Communication, Aarhus University, Jens Chr. Skous Vej 2, 8000 Aarhus C, Denmark \\ b Center for Functionally Integrative Neuroscience, Aarhus University Hospital, Nørrebrogade 44, 8000 Aarhus C, Denmark \\ c The Interacting Minds Center, Department of Culture and Society, Aarhus University, Jens Chr. Skous Vej 4, 8000 Aarhus C, Denmark \\ d Centre for Languages and Literature, Lund University, Helgonabacken 12, 22100 Lund, Sweden \\ e Department of Psychology, Hunphrey Hall, 62 Arch St., Queens University, Kingston, Ontario K7L3N6, Canada
}

\section{A R T I C L E I N F O}

\section{Article history:}

Received 14 November 2014

Accepted 18 July 2015

Available online 26 July 2015

\section{Keywords:}

fMRI

Default mode network

Memory

Narrative

\begin{abstract}
A B S T R A C T
Many everyday activities, such as engaging in conversation or listening to a story, require us to sustain attention over a prolonged period of time while integrating and synthesizing complex episodic content into a coherent mental model. Humans are remarkably capable of navigating and keeping track of all the parallel social activities of everyday life even when confronted with interruptions or changes in the environment. However, the underlying cognitive and neurocognitive mechanisms of such long-term integration and profiling of information remain a challenge to neuroscience. While brain activity is generally traceable within the short time frame of working memory (milliseconds to seconds), these integrative processes last for minutes, hours or even days. Here we report two experiments on story comprehension. Experiment I establishes a cognitive dissociation between our comprehension of plot and incidental facts in narratives: when episodic material allows for long-term integration in a coherent plot, we recall fewer factual details. However, when plot formation is challenged, we pay more attention to incidental facts. Experiment II investigates the neural underpinnings of plot formation. Results suggest a central role for the brain's default mode network related to comprehension of coherent narratives while incoherent episodes rather activate the frontoparietal control network. Moreover, an analysis of cortical activity as a function of the cumulative integration of narrative material into a coherent story reveals to linear modulations of right hemisphere posterior temporal and parietal regions. Together these findings point to key neural mechanisms involved in the fundamental human capacity for cumulative plot formation.
\end{abstract}

(c) 2015 Elsevier Inc. All rights reserved.

\section{Introduction}

Humans live complicated lives. Compared to even our nearest primate relatives, we have become reliant on hugely distributed and multileveled social and cultural structures and organization. Yet humans seem remarkably capable of navigating and keeping track of all the complex and interwoven narratives of life (ex. intrigues at the working place, international politics, fictional movies or family relations) while sustaining focus across changes in their environments. It takes sustained neurocognitive processing to engage in the extended, long-term and complex social activities and relations of human society. Although neural activity is generally traceable within the relatively short time frame of working memory, it is not clear how the brain can sustain a longerterm focus, while continuously integrating new experiential content into a coherent representational structure or situation model (Zwaan, Langston, and Graesser, 1995).

\footnotetext{
* Corresponding author at: Department for Aesthetics and Communication, Aarhus University, Jens Chr. Skous Vej 2, 8000 Aarhus C, Denmark.

E-mail address: kristian@cfin.dk (K. Tylén).
}

An example of a task that requires such sustained attention is listening to a story (Smallwood, McSpadden, and Schooler, 2008). Despite interruptions or major changes in the environment surrounding the listener, the focus can be maintained-in principle for hours-and the thematic content can be continuously updated, integrated and synthesized into a coherent cognitive model. Often the length of such interactions far exceeds the assumed limits of working memory (Baddeley, 2003). Likewise, the dynamic and continuous nature of the process lends itself uneasily to the ideas of encoding, consolidation and retrieval characteristic of most models of long-term memory (Blumenfeld and Ranganath, 2007). This kind of "slow" processing (Donald, 2007) is especially important in the comprehension of social events that extend over significant periods of time. Such events can last for minutes, hours, or days and present a challenge for the basic theory of nervous activity: how and where in the brain is the longer-term synthesis of complex stimulus material achieved?

Recent developments in the study of the brain's default mode network (DMN) can supply some initial intuitions. The default mode network comprises areas along the anterior and posterior midline, the lateral parietal cortex, prefrontal cortex, and the medial temporal lobe. 
It was originally found to activate when experimental participants were not focusing attention on the here-and-now immediacy of a task and was thus thought to reflect the 'resting brain' (Esposito et al., 2006; Raichle et al., 2001) or 'spontaneous, unconstrained thought' (Andrews-Hanna et al., 2010a, 2010b; Buckner, Andrews-Hanna, and Schacter, 2008; Harrison et al., 2008; Mason et al., 2007; Smallwood, Brown, Baird, and Schooler, 2011). However, over the last decade, researchers have been reconsidering the possible cognitive adaptations of the default mode network (Andrews-Hanna et al., 2010a, 2010b). Recent studies suggest that it is also associated with more goal-oriented and constructive processes of multi-episode integration, imagining the future and mental scene construction (Hassabis and Maguire, 2007; D. L. Schacter and Addis, 2007; Spreng, Mar, and Kim, 2009), requiring participants to attend beyond the immediacy of current perceptions (Smallwood, 2013; Smallwood et al., 2013). Furthermore, findings suggest that the default mode network flexibly couples with other networks to accomplish memory-related functional goals. For instance, it has been found that a network of areas in lateral prefrontal and parietal cortex, termed the frontoparietal control network, coactivate with the default mode network as a function of increased task demands (Meyer, Spunt, Berkman, Taylor, and Lieberman, 2012; Schacter et al., 2012; Summerfield, Hassabis, and Maguire, 2010), possibly associated with the extent to which constructive processes rely on memory (Baird, Smallwood, Gorgolewski, and Margulies, 2013).

Based on these observations, we hypothesize that the default mode network could subserve long-term, time-dependent cumulative synthesis of episodic information, henceforth plot formation. While most psychological experiments require us to momentarily focus attention on the subtleties of immediate perception within the short duration of an experimental trial, our everyday engagements in tasks, conversations and narratives only become meaningful to the extent that we can integrate and profile local information in relation to larger coherent situation models and story plots. We argue that this might be one of the main cognitive roles of the default mode network.

In the following, we address the role of the default mode network in the continuous cumulative synthesis of complex verbal stimuli into coherent plot structures. We define plot information as content crucial for the understanding of the subsequent events in a story. Often the plot comprises characters' motives or attitudes, incidents with fatal implication, or other aspects of causal relevance. In contrast, incidental facts are descriptive material that have no causal relevance for the subsequent events and often consist of characters' age, hair color, brand of car, etc. In two experiments, a behavioral and an fMRI brain imaging study, participants listened to crime stories divided into a series of short episodes. However, these episodes were interleaved with randomly chosen episodes from a set of distractor stories that did not allow for integration into a coherent plot (see Fig. 1). The experimental design allows us to study behavioral and brain components of our experience of stimuli affording long-term cumulative plot formation in contrast to stimuli that resist such integration and merely require local attention and processing.

Experiment I targeted participants' memory for plot-related information and incidental facts in the crime stories. When listening to a story, we continuously attribute differential significance to various pieces of information in anticipation of their role in the overall plot of the story (cf. 'foregrounding', Gernsbacher, Robertson, Palladino, and Werner, 2004; Talmy, 2000; Zwaan et al., 1995). To the extent that a story allows for cumulative integration of information into a coherent

Session 1

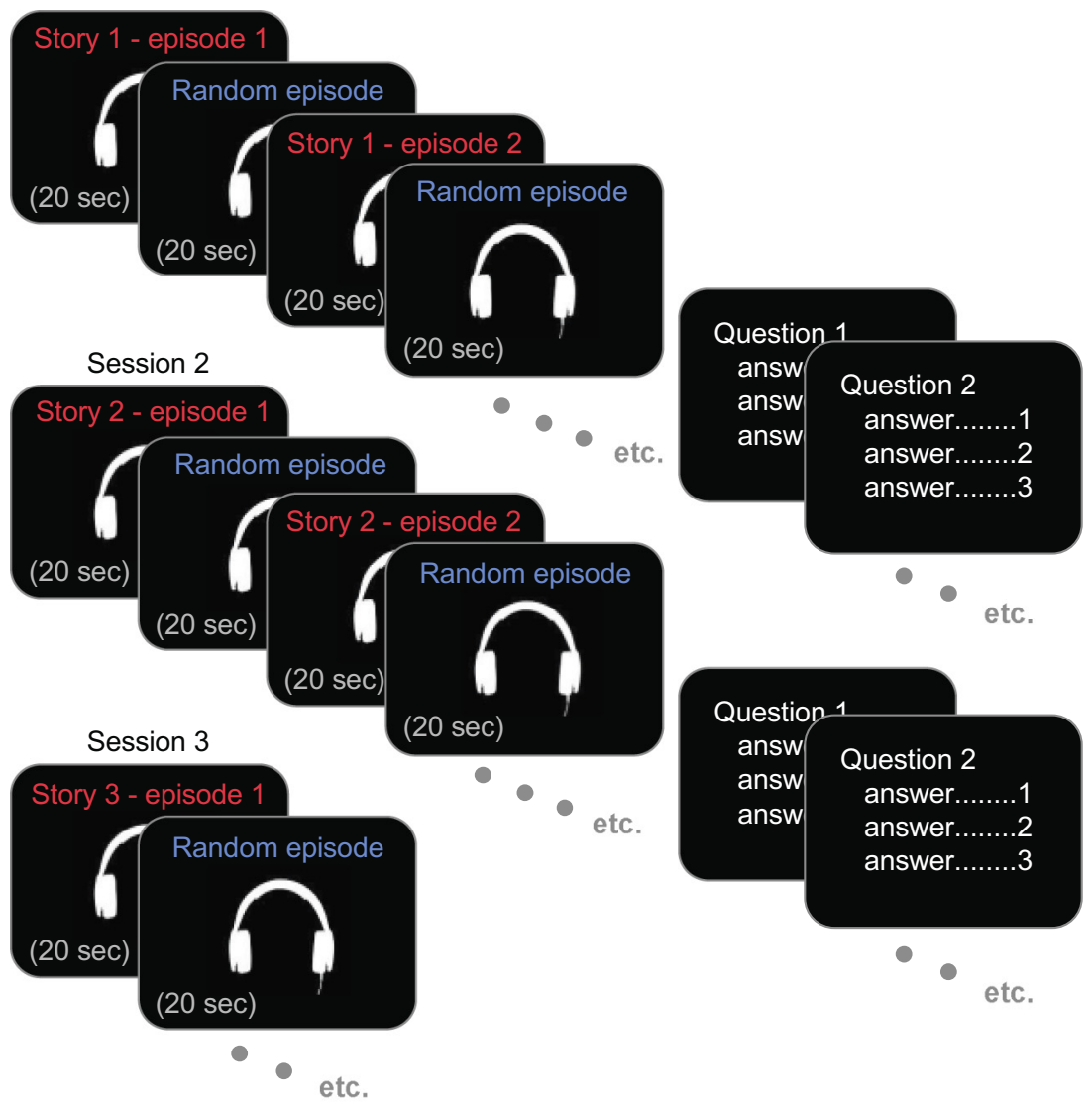

Fig. 1. Schematic depictions of the stimulus presentation design: through five sessions, participants listened to stories divided into 20 sec episodes. Six episodes together constituted a coherent story. However these episodes were interleaved with randomly chosen episodes from a set of distractor stories. 
plot structure, we predict that the recipient will tend to direct attention to key events and details while relaxing less important descriptive material ('incidental facts'). Participants will thus display better memory for plot-related material than incidental facts. However, if plot formation is challenged-i.e. if story elements do not allow for smooth integration and causal attribution as in the case of random filler episodes-we predict that the recipient cannot reliably assign differential significance to these elements with the effect that all information potentially appears equally important. In effect, participants will remember more incidental facts.

Experiment II used the same experimental design but employed functional MRI to investigate the neurophysiological basis of plot formation. This design provides a basis for assessing whether there is differential activation of the default mode network and the frontoparietal control network in response to story episodes affording long-term cumulative plot formation in contrast to episodes that resist such integration. In addition, the design allows us to identify brain areas that modulate their activity as a function of the increasing betweenepisode accumulation and profiling of information into a coherent semantic structure and to test hypotheses regarding systematic relations between performance on memory questions and brain activations.

\section{Materials and methods}

Experiment I: remembering plot relevant versus incidental information

\section{Participants}

Twenty-five adult participants ( $18 \mathrm{f} / 7 \mathrm{~m}$, aged M $23.36 \pm 2.08$ ), who had all given informed written consent, took part in the experiment in exchange for monetary compensation (DKK $200 \approx$ USD 35). Participants were primarily recruited among Danish students at Aarhus University.

\section{Stimuli and experimental design}

Stimuli were constructed from recordings of 15 short narratives of two min. duration read aloud by a male actor voice. The narratives were adopted from a series called 'Crime Story of the Week' published in 'Familie Journal' (http://www.familiejournal.dk), a popular Danish tabloid journal. They were all based on authentic criminal events evolving around murder cases and spiced up with sex, drugs and rock ' $n$ ' roll.

The participant sat in a quiet room in front of a laptop computer and listened to the stimulus narratives through headphones. The experiment was divided in five consecutive sessions consisting of a coherent story divided up in six episodes of $20 \mathrm{~s}$ (henceforth coherent stories) and interleaved with five episodes of $20 \mathrm{~s}$ randomly taken from a set of ten distractor stories (henceforth incoherent stories) (see Fig. 1 for a schematic representation of the stimulus design). A short break and a neutral sine tone indicated the shift from one part to the next. Participants were informed about the structure of the stimulus presentation.

After each session the participant was presented with eight threealternative forced choice questions assessing the participants' recall of different aspects of the stories. Questions and corresponding response options were displayed on the screen. The order of questions and position of the correct response item was randomized across trials. The participants used the keys 1-3 on the computer keyboard to submit their responses with no time constraint.

Questions were organized in a standard 2-by-2 factorial design with the factors stimulus type (coherence $+/-$ ) and question type (plot relevance $+/-$ ). Four questions thus addressed plot-relevant information (two for the coherent story and two for the distractor episodes), and four addressed incidental, descriptive facts of no relevance to the plot (again two for the coherent story and two for the distractors).

We defined plot questions as relating to information crucial for the understanding of the subsequent events in the stories. Often these were related to the characters' motives or attitudes, incidents with fatal implication, or other aspects of causal relevance. In contrast, incidental facts were defined as information with no relevance for the subsequent events or the overall plot. These questions often concerned casual facts like the profession, age or looks of the characters, the brand of car driven by a character, particular place names, family relations, etc.

\section{Analysis}

Individual accuracy scores from participants' responses to the recollection questions went into a two-way repeated-measures ANOVA. The directionality of effects was explored with planned post hoc pairedsample $t$-tests. All analyses were performed in MATLAB version 2011b (Mathworks Inc. Sherborn, MA).

\section{Experiment II: cumulative plot formation in the brain}

\section{Participants}

Twenty-four right-handed adult participants (different from the ones participating in experiment I), 12 female/12 male, aged $M=23.5$ $(S D=2.7)$ who had all given their written consent in correspondence with the requirements of the local ethical committee participated in the experiment in exchange for monetary compensation. The participants were mainly recruited among students at the humanities and health sciences at Aarhus University.

\section{Stimuli and experimental design}

The auditory stimuli and presentation design were identical to Experiment I, i.e. five sessions comprising a coherent story presented in six episodes and interleaved with five randomly chosen episodes from different narratives with an inter-stimulus interval of $2000 \mathrm{~ms}$. However, in order to optimize the paradigm for MR (e.g. not exhaust participants and complicate response recording), we replaced the elaborate multiple-choice questions with four yes/no-questions following each session. The questions were displayed at the center of the screen and participants responded by pressing one of two buttons with their right hand index and middle finger. Two questions would address coherent and two questions incoherent story episodes. The questions targeted incidental facts like characters' professions, brands of car, names of places, etc. The purpose of these questions was partly to keep participants concentrated and attentive both to coherent and incoherent stories, partly to investigate individual differences in brain activity in response to plot formation as a function of participants' behavioral responses.

\section{Scanning parameters}

We used a 3 T Siemens TIM Trio MRI system with a 12 channel head coil to acquire the $\mathrm{T} 2 *$ weighted gradient echo, echo-planar images (EPI) with Blood Oxygenation Level-Dependent (BOLD) contrast using the following parameters: echo time (TE): $27 \mathrm{~ms}$, repetition time (TR): $3020 \mathrm{~ms}$, and a flip angle of $90^{\circ}$. Four hundred fifty-seven whole-brain images were obtained over fifty-six sequential, interleaved $2 \mathrm{~mm}$ axial slices with a $2 \times 2 \mathrm{~mm}$ resolution and a field of view of $192 \times 192 \mathrm{~mm}$. In addition, we simultaneously monitored respiration and heart rate. Each scanning session took 23 min.

\section{Behavioral analysis}

Participants' response accuracies to the memory questions were averaged and compared between conditions and chance level using paired-sample t-tests, run in MATLAB.

\section{fMRI analysis}

All fMRI data analyses were conducted using SPM8 (Statistical Parametric Mapping, Wellcome Department of Imaging Neuroscience, London) implemented in MATLAB using default settings unless otherwise specified. Images were spatially realigned, normalized to the MNI template and smoothed with an isotropic $8 \mathrm{~mm}$ FWHM Gaussian kernel. Statistical analysis was conducted following a two-level general linear model approach (Penny and Holmes, 2007). On the first-level, single participant task-related BOLD responses were modeled for each subject 
by convolving condition onsets and durations with the standard hemodynamic response function and effects for each condition were estimated using a general linear model approach. The first part of the coherent stories in each session was omitted, as the conditions would only differ from the second part onwards. Two t-contrasts were estimated. The first contrast tested for the general effect of coherent over incoherent stories. A second contrast tested for the linear increase in activation level of coherent against incoherent story episodes related to the continuous accumulation of new information in a plot structure (see Fig. 3). Serial correlations were modeled explicitly using Nuisance Variable Regression (NVR), which has been shown superior to standard AR(1) modeling (Lund, Madsen, Sidaros, Luo, \& Nichols, 2006). In addition to the SPM8 default High-Pass filter (128 s cut off) we included the following covariates into the design matrix: 12 RETROICOR regressors (Glover, Li, \& Ress, 2000) based on recordings of pulse (6) and respiration (6), and 24 regressors modeling residual movement artifacts as a Volterra expansion of the movement parameters (Friston, Williams, Howard, Frackowiak, and Turner, 1996).

Contrast images from the two first-level analyses went into a second-level RFX group analysis using the one-sample $t$-test method in SPM8. The significance threshold was set to $p<.05$, FDR corrected for multiple comparisons. All corrections were carried out at the peak voxel level following the recommendations of Friston et al (1995). Functional images were overlaid with the standard SPM8 single subject high resolution T1 image.

In order to investigate individual variability in brain activation as a function of participants' responses to the memory questions, we calculated the percent difference in participants' accuracy on factual questions to coherent and incoherent story parts. The difference score was then tested against participants' contrast estimates in the peak voxel of the model testing for linear increase in brain response as a function of cumulative plot formation using the glmfit function in Matlab.

\section{Results}

\section{Experiment 1}

Participants generally performed well in the recollection task and scored an average accuracy of $74 \%(S D=16 \%)$. The ANOVA analysis revealed no significant main effects of the individual factors, but a strong 2 -way interaction, $F(1,24)=36.84, p<.0001$. Post hoc paired-sample $t$ tests revealed a number of additional effects: for coherent stories the accuracy of plot questions were significantly higher $(M=84 \%, S D=14 \%)$ than for incidental facts $(M=68 \%, S D=13 \%), t(24)=6.18, p<.0001$. For incoherent stories an opposite effect was found with higher accuracy for incidental facts $(M=78 \%, S D=16 \%)$, than for plot questions $(M=65 \%, S D=23 \%), t(24)=-3.0, p<.01$. Furthermore, the accuracy for plot questions was significantly higher for coherent $(M=84 \%, S D=$ $14 \%)$ than for in incoherent stories $(M=65 \%, S D=23 \%), t(24)=5.19$, $p<.0001$, while the accuracy for casual facts is significantly higher for incoherent $(M=78 \%, S D=16 \%)$, than for coherent stories $(M=68 \%$, $S D=13 \%$ ), $t(24)=2.49, p<.05$ (see Fig. 2 ).

\section{Experiment 2}

Participants responded significantly less accurately to yes/no questions about incidental facts for coherent story episodes than incoherent, $t(1,24)=-3.30, p<.005$. Responses for coherent story episodes were at chance level, $t(1,24)=0.53, p=n s$, while responses for incoherent story episodes were significantly over chance, $t(1,24)=6.04, p<.0001$.

Relative to incoherent story episodes, coherent story episodes yielded significant activations of posterior cingulate gyrus/precuneus, anterior prefrontal cortex, orbitofrontal cortex, right hippocampus and right caudate nucleus, as well as large areas of the temporal lobes extending from the temporal poles to the temporo-parietal junction

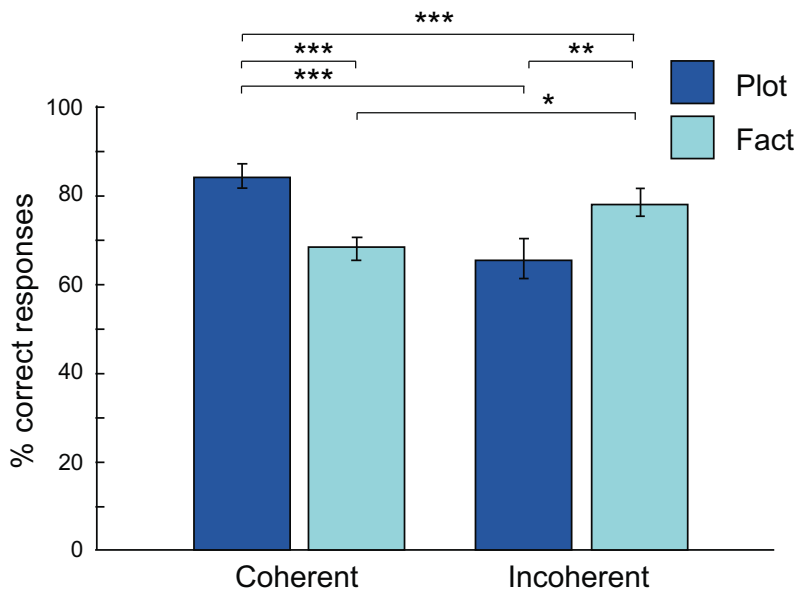

Fig. 2. Results from experiment 1, testing the memory for incidental facts and plot-related information in coherent and incoherent story episodes. Participants generally remembered more plot-related information from coherent episodes while they remembered more incidental facts from incoherent episodes. Error bars represent standard error of the mean. ${ }^{* * *} \mathrm{p}<.0001,{ }^{* *} \mathrm{p}<.01,{ }^{*} \mathrm{p}<.05$.

bilaterally with peaks in right temporal pole and left middle temporal lobe. Many of these sites are considered part of the default mode network (see Table 1A and Fig. 3a for details).

Table 1

fMRI activations sites and z-scores for A: coherent > incoherent episodes, B: incoherent > coherent episodes, C: cumulative plot formation for coherent > incorherent, D: cumulative plot formation for coherent $>$ baseline. All contrasts are thresholded at $\mathrm{p}<.05$, FDR corrected for multiple comparisons.

\begin{tabular}{|c|c|c|c|c|}
\hline \multirow[t]{2}{*}{ Putative anatomical regions } & \multirow[t]{2}{*}{ Z-scores } & \multicolumn{3}{|c|}{ Coordinates (MNI) } \\
\hline & & $\mathrm{x}$ & $\mathrm{y}$ & $\mathrm{z}$ \\
\hline \multicolumn{5}{|l|}{ A: Coherent > incoherent episodes } \\
\hline Superior temporal pole (R) & 6.35 & 52 & 10 & -20 \\
\hline Middle temporal lobe (L) & 5.85 & -52 & -16 & -8 \\
\hline Posterior cingulate/precuneus (R) & 5.48 & 2 & -56 & 28 \\
\hline $\mathrm{mPFC}(\mathrm{L})$ & 5.14 & -6 & 52 & 42 \\
\hline Orbito frontal cortex (L) & 5.14 & -2 & 58 & -12 \\
\hline Post central gyrus (L) & 5.05 & -60 & -12 & 38 \\
\hline IFG opercularis ( $R$ ) & 4.70 & 54 & 14 & 22 \\
\hline Hippocampus (R) & 4.55 & 22 & -8 & -16 \\
\hline Caudate $(\mathrm{R})$ & 4.35 & 20 & 12 & 10 \\
\hline \multicolumn{5}{|l|}{ B: Incoherent > coherent episodes } \\
\hline Anterior cingulate gyrus $(\mathrm{R})$ & 6.53 & 4 & 28 & 34 \\
\hline Middle frontal/frontopolar cortex (R) & 6.22 & 34 & 48 & 28 \\
\hline Inferior parietal lobe $(\mathrm{R})$ & 6.22 & 52 & -44 & 50 \\
\hline Inferior parietal lobe $(\mathrm{L})$ & 5.93 & -56 & -42 & 52 \\
\hline $\operatorname{DLPFC}(\mathrm{R})$ & 5.72 & 46 & 40 & 30 \\
\hline DLPFC (L) & 4.47 & -32 & 42 & 28 \\
\hline Inferior temporal lobe $(\mathrm{R})$ & 5.56 & 58 & -36 & -16 \\
\hline Anterior insula $(\mathrm{R})$ & 5.10 & 36 & 16 & 0 \\
\hline Anterior insula (L) & 4.82 & -36 & 14 & 2 \\
\hline Superior frontal gyrus/frontal eye fields ( $R$ ) & 4.50 & 26 & 8 & 64 \\
\hline Superior frontal gyrus/frontal eye fields (L) & 3.61 & -16 & 6 & 64 \\
\hline Inferior temporal lobe $(\mathrm{L})$ & 4.26 & -58 & -58 & -10 \\
\hline \multicolumn{5}{|c|}{ C: Cumulative plot-formation-coherent $>$ incoherent } \\
\hline Posterior cingulate/precuneus ( $\mathrm{R})$ & 5.66 & 6 & -64 & 30 \\
\hline Posterior cingulate/precuneus (L) & 4.12 & -4 & -60 & 40 \\
\hline Superior temporal sulcus $(\mathrm{R})$ & 5.18 & 62 & -44 & 12 \\
\hline Middle temporal gyrus $(\mathrm{R})$ & 4.49 & 50 & -2 & -20 \\
\hline Middle frontal/frontopolar cortex (L) & 4.03 & -50 & 24 & 40 \\
\hline Precentral gyrus (L) & 3.99 & -42 & 10 & 46 \\
\hline Superior frontal lobe $(\mathrm{R})$ & 3.98 & 30 & 48 & 8 \\
\hline Middle temporal lobe (L) & 3.80 & -50 & -50 & 8 \\
\hline \multicolumn{5}{|l|}{ D: Cumulative plot-formation-coherent $>$ baseline } \\
\hline Superior temporal lobe $(\mathrm{L})$ & 7.37 & -64 & -16 & 2 \\
\hline Superior temporal lobe $(\mathrm{R})$ & 6.72 & 60 & -22 & 2 \\
\hline Postcentral gyrus (R) & 5.06 & 40 & -28 & 58 \\
\hline
\end{tabular}


a: Coherent > incoherent story episodes
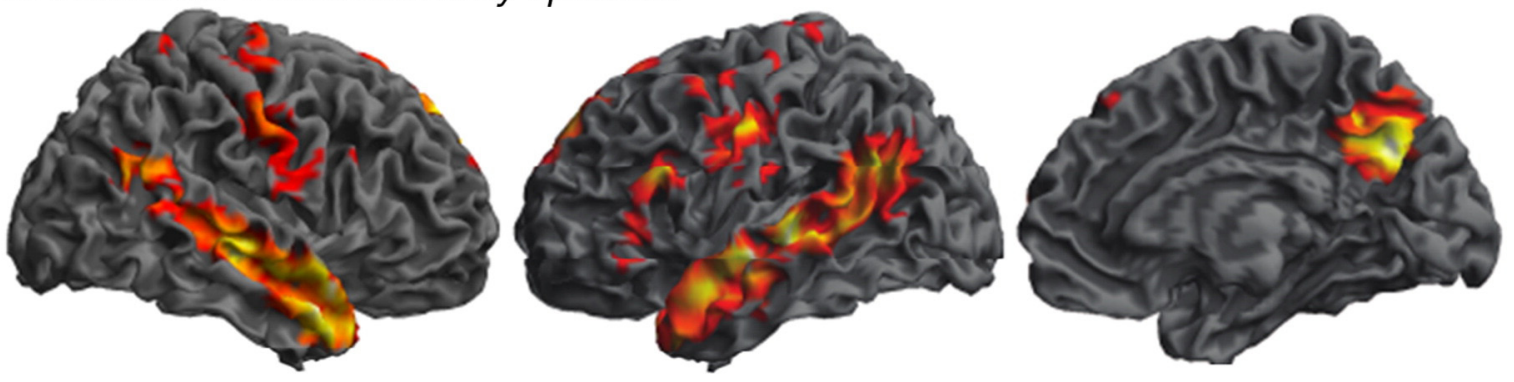

b: Incoherent > coherent story episodes
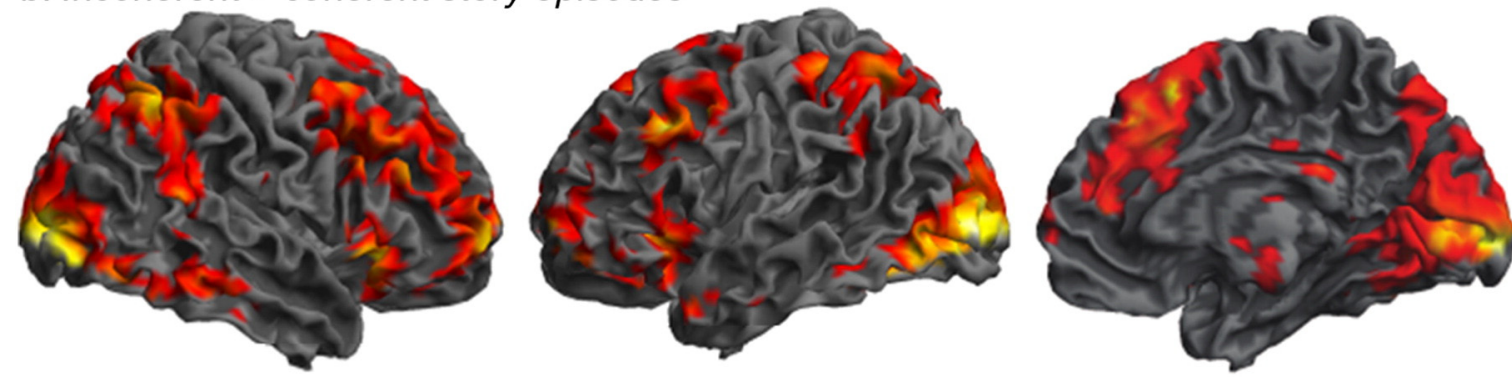

Fig. 3. fMRI results. a: all coherent events contrasted to all incoherent events gives activation of the default mode network, in particular posterior cingulate gyrus/precuneus, anterior prefrontal cortex, right hippocampus and middle temporal cortices. b: All incoherent events contrasted to all coherent events activated the frontoparietal control network, in particular the frontopolar, the anterior insula bilaterally, and the inferior parietal lobes bilaterally $(\mathrm{p}<.05$, FDR corrected for multiple comparisons).

In contrast, incoherent story episodes yielded stronger activations in the frontopolar cortex, dorsolateral prefrontal cortex bilaterally, the anterior cingulate gyrus, superior frontal gyrus/frontal eye fields bilaterally, the anterior insula bilaterally, the inferior parietal lobes bilaterally, and the inferior temporal lobes bilaterally. Areas that have putatively been suggested part of a frontoparietal control network (see Table 1B and Fig. 3b).
The parametric model assuming linearly increasing activity as a function of cumulative plot formation over coherent versus incoherent episodes was found significant in right hemisphere middle and posterior superior temporal sulcus/angular gyrus, posterior cingulate/ precuneus, right superior frontal lobe and the precentral gyrus bilaterally. The opposite contrast yielded no significant activations (see Table $1 \mathrm{C}$ and Fig. 4).

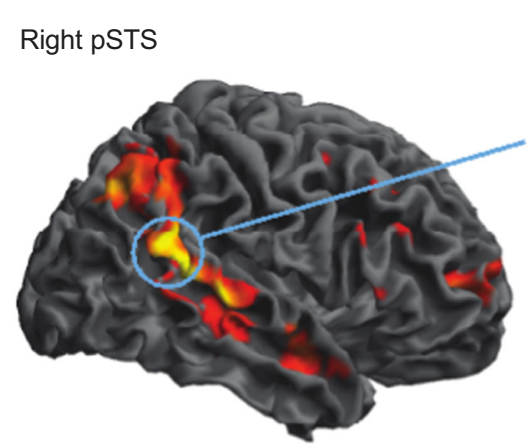

Right precuneus

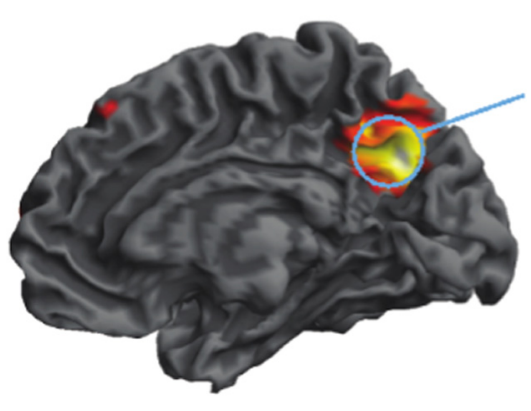

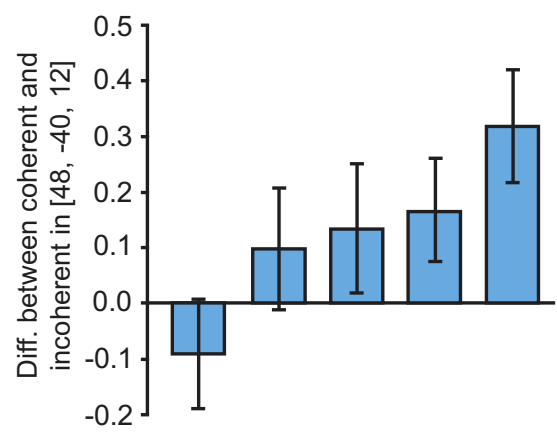

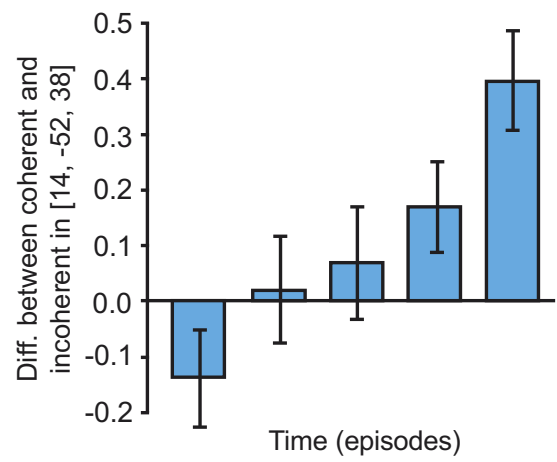

Fig. 4. Cumulative plot formation as tested by a parametric model assuming linear increase in functional activity over coherent episodes. The Y-axis represents pairwise differences in contrast estimates of target voxels between coherent and incoherent episodes at each time point. Activity was primarily found in right hemisphere middle and posterior superior temporal sulcus and the precuneus ( $\mathrm{p}<.05$, FDR corrected for multiple comparisons). Error bars represent 95\% confidence intervals. 
When tested against a neutral baseline of unmodelled events, the plot formation model was found significant in superior temporal sulci bilaterally and the right postcentral gyrus, see Table 1D.

Variability in participants' default mode network response to cumulative plot formation was significantly predicted by individual differences in their responses to the memory questions. Participants who were more accurate in their responses to fact questions about incoherent than coherent stories also had stronger contrast estimates in the posterior superior temporal sulcus, slope $=1.99, \mathrm{p}<0.05$ (see Fig. 5).

\section{Discussion}

In everyday life, we constantly navigate in and out of multiple parallel narratives: the ongoing projects and conversations at work, all the daily and weekly cycles of family life, our favorite television shows and bedtime novels. Even though these contexts present themselves in series of interrupted segments often scattered over huge time periods, they tend to constitute perfectly well-formed and coherent experiences in conscious memory. This ability to cumulate, synthesize and profile fragmented information into coherent narratives seems an absolutely central key in understanding the complex and distributed character of human cognition and social organization (Donald, 2007).

In this study we investigated the behavioral and neural processes related to cumulative synthesis of information associated with the reception of episodic content in such interleaved and scattered event structures.

\section{Recalling episodic content: plot versus incidental facts}

In the first experiment, we found that the extent to which individual story episodes allow for cumulative plot formation affects the participant's attention to-and recall of-different types of information. When presented with episodes that afforded integration into a coherent story, participants remembered plot-relevant details such as characters' motives, dispositions and key events. However, they had problems recalling casual descriptive details such as characters' profession, age, brand of car etc. when these were of little or no importance to the overall plot. The opposite effect was found when participants were presented with story episodes that did not relate to each other to form coherent narratives. Here, as expected, participants generally faired worse on plot-related questions: it is hard to pick up information crucial

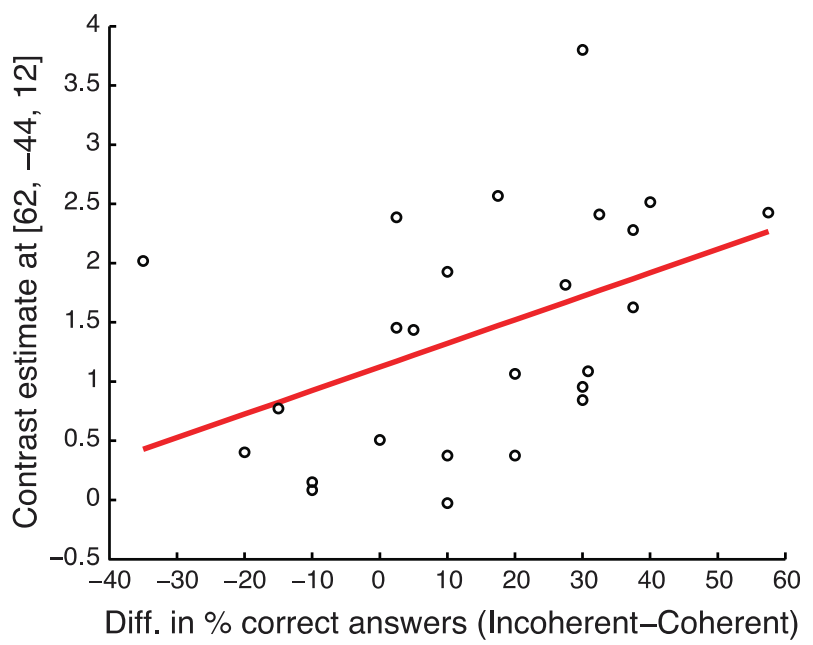

Fig. 5. The relation between behavioral responses and activity in the temporal DMN. The $\mathrm{x}$-axis reflects individual participants' differences in accuracy on memory questions concerning coherent and incoherent stories. The y-axis reflects their contrast estimates from the right pSTS in the test of linear increase as a function of cumulative plot formation. The larger the difference in accuracy, the stronger the contrast estimate. for the plot when plot formation is severely interrupted. However, in this condition participants showed enhanced memory for incidental, descriptive content. The behavioral testing recorded in the scanner in relation to Experiment II replicated these findings (although, here we asked yes/no questions and only concerning incidental facts): Participants showed significantly greater recall for incidental facts when presented with incoherent story episodes than coherent story episodes. In fact, participants performed at chance when tested on coherent stories, suggesting that incidental details get profoundly backgrounded when plot construction is made possible. Together the findings suggest that the continuous construction of a narrative plot works to profile certain information as salient foreground crucial for understanding the overall course of the story, while other information (incidental facts) come to merely constitute the background (Gernsbacher et al., 2004). Our observations resonate with studies on reading and mind wandering (Smallwood et al., 2008). Here, mind wandering was found particularly detrimental to understanding if it occurred at critical plot-related moments during the reading of narratives. When plot formation is challenged, participants suddenly lack an important criterion of relevance, making all information equally salient. In our study, participants' behavior seems indicative of an implicit strategy to remember more casual details in the hope that subsequent events will allow them to retrospectively reconstruct the plot.

\section{Longer-term integration: a role for the default mode network?}

Experiment II relied on fMRI to investigate brain structures subserving long-term, cumulative plot formation. Contrasting coherent story episodes against incoherent episodes, we observed strong activations in areas of the default mode network including $\mathrm{MPFC}$, posterior cingulate, right hippocampus, caudate nucleus and medial temporal gyrus extending to temporal poles and angular gyrus/TPJ. These areas have previously been found to consistently activate in studies on semantic integration. Relying on a between-subject correlational fMRI technique, Hasson and colleagues (Hasson, Yang, Vallines, Heeger, and Rubin, 2008; Lerner, Honey, Silbert, and Hasson, 2011) presented participants with narrative stimuli either scrambled at the level of words, sentences, or paragraphs and contrasted them with processes associated with a full seven-minute narrative. As a function of increasingly larger windows of semantic integration, they found enhanced activity of $\mathrm{mPFC}$, posterior cingulate/precuneus as well as posterior superior temporal areas bilaterally. Their findings thus suggest a hierarchical cortical organization with longer time scales of integration selectively recruiting areas of the default mode network. While in these studies the cognitive implications are not directly assessed, other studies point to the constructive and cumulative character of such long-term semantic integration. Through a series of studies, Hassabis and Maguire investigated the modulation of the default mode network in tasks that required participants to construct coherent mental scenes from multiple sequentially presented pieces of information (Hassabis and Maguire, 2007, 2009). Interestingly, a recent study found that in these contexts, the activation profile in posterior cortical regions (e.g. angular gyrus and intra-parietal sulcus) followed the semantic saturation of a scene as reported by participants (Summerfield et al., 2010). This suggests that these integration processes are modulated by the structure of semantic content rather than mere quantity of stimulus integrated.

The default mode network has also been consistently found in studies on prospective cognition, that is, situations where we draw upon memory to simulate future events (Andrews-Hanna et al., 2010a, 2010b; Gerlach, Spreng, Gilmore, and Schacter, 2011; D. L. Schacter and Addis, 2007; D.L. Schacter et al., 2012). In these cases, the default mode network is involved in the continuous updating of prospective mental models to guide attention and action in anticipatory ways.

While semantic integration and prospective cognition have been considered different functions associated with the default mode network, we argue that they both form key elements of cumulative 
plot formation: a process that integrates new information in the context of an ongoing situation or narrative to construct and continuously update the plot, which provides a prospective model of the overall story line. As suggested by our behavioral findings, such models in turn provide a criterion for differential distribution of attention in the narratives with the consequence that some information come to constitute foreground while other information is pushed to the background. Moreover, adding to existing evidence, the present findings indicate how the default mode network is involved in integrative, constructive, and prospective processes spanning even fragmented and interrupted experiences over larger time intervals.

\section{Cumulative plot formation and the right middle and posterior temporal gyrus}

To test which brain areas would modulate their activity as a function of the continuous cumulative integration of information into a coherent story, we applied a parametric model assuming a linear increase in activation through coherent story episodes, in contrast to incoherent story episodes where such semantic accumulation is not possible. Faced with the task of long-term maintenance of existing content, and integration of new episodic content into one coherent semantic representation, we hypothesized that some brain areas would continuously up-regulate their activity over the course of a coherent narrative. Again, this analysis yielded activation in the default mode network, however, this time only in a particular subset of areas including posterior cingulate/precuneus, right middle and posterior temporal gyrus, extending towards TPJ. Although laterality effects are not directly tested in these analyses, we notice that primarily right hemisphere posterior temporal regions seem to continuously increase activity in response to the cumulative development of a narrative plot line. Modulation of right temporal areas in response to complex narrative integration has previously been observed (Xu, Kemeny, Park, Frattali, and Braun, 2005), although not in the context of continuous cumulative plot formation.

This result coincides with the classic view that the right hemisphere is involved in processing of global aspects of linguistic contents (Poeppel, 2003; St George, Kutas, Martinez, and Sereno, 1999), also supported by right hemisphere lesion studies (Weed, McGregor, Feldbaek Nielsen, Roepstorff, and Frith, 2010). In addition to the general observation of a right hemisphere network for cumulative plot formation, explorative mapping of contrast estimates revealed differential underlying sources of contrast differences within the network. While contrastive patterns in middle and posterior temporal regions were driven by increase in activity over coherent episodes, activity in the precuneus was rather driven by gradual decrease of activity in response to incoherent episodes. This indicates that areas in closer proximity to primary perceptual regions are mainly responsible for the cumulative buffering of information potentially relevant for the evolving plotline. The posterior cingulate/precuneus-in turn-maintained a stable level of activation throughout the coherent episodes in contrast to a gradual drop in activity in response to incoherent episodes. This indicates that this node might be responsible for the long-term constructive integration process, which relies on the buffered information from temporal regions (Daselaar et al., 2008).

\section{The frontoparietal control network and incoherent episodic content}

Interestingly, when contrasting incoherent episodes against coherent ones we find strong activations in a network, comprising, among others, the frontopolar cortex, the anterior cingulate, dorsolateral prefrontal cortex, anterior insula and lateral parietal cortex. This network, sometimes referred to as the frontoparietal control network (Vincent, Kahn, Snyder, Raichle, and Buckner, 2008), has been suggested to have a mediating role between the default mode network primarily concerned with self-generated thought and the dorsal attention network oriented towards external environmental stimuli (Fox et al., 2005; Vincent et al., 2008). Not unlike the default mode network, the frontoparietal control network has been associated with cognitive tasks requiring maintenance or integration of information over sustained periods of time (Dosenbach et al., 2006; Velanova et al., 2003; Yarkoni et al., 2005). However, the frontoparietal control network has also been observed to activate in studies requiring participants to integrate working memory processes with enhanced attentional demands to simultaneously consider multiple conflicting contingencies (Crone, Wendelken, Donohue, and Bunge, 2006; Koechlin, Basso, Pietrini, Panzer, and Grafman, 1999; Kroger et al., 2002). Along similar lines, activity in one of the central nodes in this network-the frontopolar cortex-is consistently modulated by the degree of uncertainty when multiple alternative interpretations are simultaneously present (Yoshida and Ishii, 2006), or when participants depart from one interpretation to check alternative ones (Daw, O'Doherty, Dayan, Seymour, and Dolan, 2006; Koechlin and Hyafil, 2007).

In the present study, we found the frontoparietal network to activate when participants were confronted with individual story episodes resisting integration with preceding episodes. In these cases, participants could not rely on preceding episodes to offer a model by which new information could be integrated and profiled, and in effect each single episode had to be individually modeled. This is likely to exert increased attentional demands on participants: without preceding context, there are no criteria by which to differentially distribute attention to the relevant details and all information may appear equally important. This is supported by the behavioral finding that participants tended to have better recollection for otherwise insignificant casual facts in this condition.

\section{Relations between behavior and brain response}

Interestingly, individual variability in participants' DMN responses was predicted by their behavioral responses to the memory questions in the scanner. Those participants who had very different levels of accuracy on questions to coherent and incoherent stories (lower on coherent) were also those who had higher contrast estimates in right pSTS to the parametric model assuming linear increase in brain activity over coherent story parts. Following predictions from experiment 1 , activity in temporal areas of the DMN is modulated by participants' attention to various aspects of the stories: When presented to story content that affords plot-formation, participants form representations at a longer-term, superordinate level, however at the expense of attention to factual details. Participants who paid less attention to incidental facts in coherent stories than in incoherent stories had better fits to the model in temporal parts of the DMN, than those who attended more evenly to facts in both coherent and incoherent stories. This is a strong indication that modulations in DMN activity is indeed systematically linked to functional cognitive processes of long-term integration and synthesis of episodic content, that is, cumulative plot formation.

\section{General discussion}

Since the first discovery of the default mode network by Raichle and colleagues more than ten years ago, researchers have wondered about its possible cognitive adaptations. From initial ideas about resting states and mind wandering, recent studies have suggested the engagement of this network in more goal-oriented cognitive processes of for instance semantic integration, scene construction, and simulation of the future. In this study we present behavioral and neurocognitive evidence that the default mode network is involved in a process of cumulative plot formation, possibly drawing on several of these other components. Cumulative plot formation allows us to keep track of even scattered episodic content and integrating it into larger scale coherent semantic clusters such as for instance narratives. This process involves three 
central components: 1) integration of episodic content over larger time intervals, 2) prospective modeling of this information into a coherent plot line, 3 ) the profiling of information in foreground and background relative to the plot to guide attention to future episodes. We found that when a series of episodes afford integration into a coherent story line, people remember information relevant for the overall plot while they do not recall incidental facts (descriptive details). In these cases we find brain activity in the default mode and in particular in right posterior temporal areas and in precuneus. In contrast, when episodic content resists such long-term integration, people do not have a working criterion for selective attention and thus tend to remember more incidental facts. In these cases we observe activations of the frontoparietal control network, a network often associated with the resolution of conflicting alternatives and tasks requiring increased attentional demands. Together our findings point to the default mode as playing a key role in the uniquely human capacity for long-term integration and synthesis of episodic content associated with our constant navigation of multiple, distributed and multileveled social and cultural contexts (Donald, 2007).

\section{Acknowledgments}

The authors would like to thank Karsten Olsen and Dan Bang for their valuable contributions in early phases of this study. The study was supported by the Danish Council for Independent Research's (903906) grant Joint Diagrammatical Reasoning in Language and the EUROcores program, Digging the Roots of Understanding (DRUST).

\section{References}

Andrews-Hanna, J.R., Reidler, J.S., Huang, C., Buckner, R.L., 2010a. Evidence for the default network's role in spontaneous cognition. J. Neurophysiol. 104 (1), 322-335. http://dx. doi.org/10.1152/jn.00830.2009.

Andrews-Hanna, J.R., Reidler, J.S., Sepulcre, J., Poulin, R., Buckner, R.L., 2010b. Functionalanatomic fractionation of the brain's default network. Neuron 65 (4), 550-562. http://dx.doi.org/10.1016/j.neuron.2010.02.005.

Baddeley, A., 2003. Working memory: looking back and looking forward. Nat. Rev. Neurosci. 4 (10), 829-839. http://dx.doi.org/10.1038/nrn1201.

Baird, B., Smallwood, J., Gorgolewski, K.J., Margulies, D.S., 2013. Medial and lateral networks in anterior prefrontal cortex support metacognitive ability for memory and perception. J. Neurosci. 33 (42), 16657-16665.

Blumenfeld, R.S., Ranganath, C., 2007. Prefrontal cortex and long-term memory encoding: an integrative review of findings from neuropsychology and neuroimaging. Neuroscientist 13 (3), 280-291. http://dx.doi.org/10.1177/1073858407299290.

Buckner, R.L., Andrews-Hanna, J.R., Schacter, D.L., 2008. The brain's default network: anatomy, function, and relevance to disease. Ann. N. Y. Acad. Sci. 1124, 1-38. http://dx. doi.org/10.1196/annals.1440.011.

Crone, E.A., Wendelken, C., Donohue, S.E., Bunge, S.A., 2006. Neural evidence for dissociable components of task-switching. Cereb. Cortex 16 (4), 475-486. http://dx.doi.org/ 10.1093/cercor/bhi127.

Daselaar, S.M., Rice, H.J., Greenberg, D.L., Cabeza, R., LaBar, K.S., Rubin, D.C., 2008. The spatiotemporal dynamics of autobiographical memory: neural correlates of recall, emotional intensity, and reliving. Cereb. Cortex 18 (1), 217-229. http://dx.doi.org/ 10.1093/cercor/bhm048.

Daw, N.D., O'Doherty, J.P., Dayan, P., Seymour, B., Dolan, R.J., 2006. Cortical substrates for exploratory decisions in humans. Nature 441 (7095), 876-879. http://dx.doi.org/10. 1038/nature04766.

Donald, M., 2007. The slow process: a hypothetical cognitive adaptation for distributed cognitive networks. J. Physiol. Paris 101 (4-6), 214-222. http://dx.doi.org/10.1016/j. jphysparis.2007.11.006

Dosenbach, N.U., Visscher, K.M., Palmer, E.D., Miezin, F.M., Wenger, K.K., Kang, H.C., Petersen, S.E., 2006. A core system for the implementation of task sets. Neuron 50 (5), 799-812. http://dx.doi.org/10.1016/j.neuron.2006.04.031.

Esposito, F., Bertolino, A., Scarabino, T., Latorre, V., Blasi, G., Popolizio, T., Di Salle, F., 2006. Independent component model of the default-mode brain function: assessing the impact of active thinking. Brain Res. Bull. 70 (4-6), 263-269. http://dx.doi.org/10.1016/j. brainresbull.2006.06.012.

Fox, M.D., Snyder, A.Z., Vincent, J.L., Corbetta, M., Van Essen, D.C., Raichle, M.E., 2005. The human brain is intrinsically organized into dynamic, anticorrelated functional networks. Proc. Natl. Acad. Sci. U. S. A. 102 (27), 9673-9678. http://dx.doi.org/10.1073/ pnas.0504136102

Friston, K.J., Holmes, A.P., Worsley, K.J., Poline, J.B., Frith, C.D., Frackowiak, R.S., 1995. Statistical parametric maps in functional imaging: a general linear approach. Hum. Brain Mapp. 2, 189-210

Friston, K.J., Williams, S., Howard, R., Frackowiak, R.S., Turner, R., 1996. Movement-related effects in fMRI time-series. Magn. Reson. Med. 35 (3), 346-355.
Gerlach, K.D., Spreng, R.N., Gilmore, A.W., Schacter, D.L., 2011. Solving future problems: default network and executive activity associated with goal-directed mental simulations. NeuroImage 55 (4), 1816-1824. http://dx.doi.org/10.1016/j.neuroimage.2011. 01.030 .

Gernsbacher, M.A., Robertson, R.R., Palladino, P., Werner, N.K., 2004. Managing mental representations during narrative comprehension. Discourse Process. 37 (2), 145-164.

Glover, G.H., Li, T.Q., Ress, D., 2000. Image-based method for retrospective correction of physiological motion effects in fMRI: RETROICOR. Magn. Reson. Med. 44 (1), 162-167.

Harrison, B.J., Pujol, J., López-Solà, M., Hernández-Ribas, R., Deus, J., Ortiz, H., Cardoner, N 2008. Consistency and functional specialization in the default mode brain network. Proc. Natl. Acad. Sci. 105 (28), 9781.

Hassabis, D., Maguire, E.A., 2007. Deconstructing episodic memory with construction. Trends Cogn. Sci. 11 (7), 299-306. http://dx.doi.org/10.1016/j.tics.2007.05.001.

Hassabis, D., Maguire, E.A., 2009. The construction system of the brain. Philos. Trans. R. Soc. Lond. B Biol. Sci. 364 (1521), 1263-1271. http://dx.doi.org/10.1098/rstb.2008. 0296.

Hasson, U., Yang, E., Vallines, I., Heeger, D.J., Rubin, N., 2008. A hierarchy of temporal receptive windows in human cortex. J. Neurosci. 28 (10), 2539-2550. http://dx.doi. org/10.1523/JNEUROSCI.5487-07.2008.

Koechlin, E., Hyafil, A., 2007. Anterior prefrontal function and the limits of human decision-making. Science 318 (5850), 594-598. http://dx.doi.org/10.1126/science. 1142995

Koechlin, E., Basso, G., Pietrini, P., Panzer, S., Grafman, J., 1999. The role of the anterior prefrontal cortex in human cognition. Nature 399 (6732), 148-151. http://dx.doi.org/10. 1038/20178.

Kroger, J.K., Sabb, F.W., Fales, C.L., Bookheimer, S.Y., Cohen, M.S., Holyoak, K.J., 2002. Recruitment of anterior dorsolateral prefrontal cortex in human reasoning: a parametric study of relational complexity. Cereb. Cortex 12 (5), 477-485.

Lerner, Y., Honey, C.J., Silbert, L.J., Hasson, U., 2011. Topographic mapping of a hierarchy of temporal receptive windows using a narrated story. J. Neurosci. 31 (8), 2906-2915. http://dx.doi.org/10.1523/JNEUROSCI.3684-10.2011.

Lund, T.E., Madsen, K.H., Sidaros, K., Luo, W.L., Nichols, T.E., 2006. Non-white noise in fMRI: does modelling have an impact? NeuroImage 29 (1), 54-66.

Mason, M.F., Norton, M.I., Van Horn, J.D., Wegner, D.M., Grafton, S.T., Macrae, C.N., 2007 Wandering minds: the default network and stimulus-independent thought. Science 315 (5810), 393-395. http://dx.doi.org/10.1126/science.1131295.

Meyer, M.L., Spunt, R.P., Berkman, E.T., Taylor, S.E., Lieberman, M.D., 2012. Evidence for social working memory from a parametric functional MRI study. Proc. Natl. Acad. Sci. U. S. A. 109 (6), 1883-1888. http://dx.doi.org/10.1073/pnas.1121077109.

Penny, W., Holmes, A.P., 2007. Random effects analysis. In: Friston, K.J., Ashburner, J., Kiebel, S., Nichols, T., Penny, W. (Eds.), Statistical Parametric Mapping: The Analysis of Functional Brain Images. Academic Press, London.

Poeppel, D., 2003. The analysis of speech in different temporal integration windows: cerebral lateralization as 'asymmetric sampling in time'. Speech Comm. 41 (1) 245-255. http://dx.doi.org/10.1016/S0167-6393(02)00107-3.

Raichle, M.E., MacLeod, A.M., Snyder, A.Z., Powers, W.J., Gusnard, D.A., Shulman, G.L 2001. A default mode of brain function. Proc. Natl. Acad. Sci. U. S. A. 98 (2) 676-682. http://dx.doi.org/10.1073/pnas.98.2.676.

Schacter, D.L., Addis, D.R., 2007. The cognitive neuroscience of constructive memory: remembering the past and imagining the future. Philos. Trans. R. Soc. Lond. Ser. B Biol. Sci. 362 (1481), 773-786. http://dx.doi.org/10.1098/rstb.2007.2087.

Schacter, D.L., Addis, D.R., Hassabis, D., Martin, V.C., Spreng, R.N., Szpunar, K.K., 2012. The future of memory: remembering, imagining, and the brain. Neuron 76 (4), 677-694.

Smallwood, J., 2013. Distinguishing how from why the mind wanders: a processoccurrence framework for self-generated mental activity. Psychol. Bull. 139 (3) 519-535. http://dx.doi.org/10.1037/a0030010.

Smallwood, J., McSpadden, M., Schooler, J.W., 2008. When attention matters: the curious incident of the wandering mind. Mem. Cogn. 36 (6), 1144-1150. http://dx.doi.org/10. 3758/MC.36.6.1144.

Smallwood, J., Brown, K., Baird, B, Schooler, J.W., 2011. Cooperation Between the Default Mode Network and the Frontal-Parietal Network in the Production of an Internal Train of Thought. Brain research.

Smallwood, J., Tipper, C., Brown, K., Baird, B., Engen, H., Michaels, J.R., Schooler, J.W., 2013. Escaping the here and now: evidence for a role of the default mode network in perceptually decoupled thought. NeuroImage 69, 120-125. http://dx.doi.org/10.1016/j. neuroimage.2012.12.012

Spreng, R.N., Mar, R.A., Kim, A.S.N., 2009. The common neural basis of autobiographical memory, prospection, navigation, theory of mind, and the default mode: a quantitative meta-analysis. J. Cogn. Neurosci. 21 (3), 489-510.

St George, M., Kutas, M., Martinez, A., Sereno, M.I., 1999. Semantic integration in reading: engagement of the right hemisphere during discourse processing. Brain 122 (Pt 7) $1317-1325$

Summerfield, J.J., Hassabis, D., Maguire, E.A., 2010. Differential engagement of brain regions within a 'core' network during scene construction. Neuropsychologia 48 (5), 1501-1509. http://dx.doi.org/10.1016/j.neuropsychologia.2010.01.022.

Talmy, L., 2000. Toward a Cognitive Semantics. MIT Press, Cambridge, Mass.

Velanova, K., Jacoby, L.L., Wheeler, M.E., McAvoy, M.P., Petersen, S.E., Buckner, R.L., 2003. Functional-anatomic correlates of sustained and transient processing components engaged during controlled retrieval. J. Neurosci. 23 (24), 8460-8470.

Vincent, J.L., Kahn, I., Snyder, A.Z., Raichle, M.E., Buckner, R.L., 2008. Evidence for a frontoparietal control system revealed by intrinsic functional connectivity J. Neurophysiol. 100 (6), 3328-3342. http://dx.doi.org/10.1152/jn.90355.2008.

Weed, E., McGregor, W., Feldbaek Nielsen, J., Roepstorff, A., Frith, U., 2010. Theory of mind in adults with right hemisphere damage: what's the story? Brain Lang. 113 (2), 65-72. http://dx.doi.org/10.1016/j.bandl.2010.01.009. 
Xu, J., Kemeny, S., Park, G., Frattali, C., Braun, A., 2005. Language in context: emergent features of word, sentence, and narrative comprehension. Neurolmage 25 (3), 1002-1015. Yarkoni, T., Gray, J.R., Chrastil, E.R., Barch, D.M., Green, L., Braver, T.S., 2005. Sustained neural activity associated with cognitive control during temporally extended decision making. Brain Res. Cogn. Brain Res. 23 (1), 71-84. http://dx.doi.org/10.1016/j. cogbrainres.2005.01.013.
Yoshida, W., Ishii, S., 2006. Resolution of uncertainty in prefrontal cortex. Neuron 50 (5), 781-789. http://dx.doi.org/10.1016/j.neuron.2006.05.006.

Zwaan, R.A., Langston, M.C., Graesser, A.C., 1995. The construction of situation models in narrative comprehension: an event-indexing model. Psychol. Sci. 6 (5), 292-297. http://dx.doi.org/10.2307/40063035. 